Proceedings of the Edinburgh Mathematical Society (2006) 49, 367-381 (C)

DOI:10.1017/S0013091504000628 Printed in the United Kingdom

\title{
ON CONTINUOUS AND ADJOINT MORPHISMS BETWEEN NON-COMMUTATIVE PRIME SPECTRA
}

\author{
EDWARD S. LETZTER \\ Department of Mathematics, Wachman Hall, 1805 North Broad Street, \\ Temple University, Philadelphia, PA 19122, USA \\ (letzter@math.temple.edu)
}

(Received 8 July 2004)

\begin{abstract}
We study topological properties of the correspondence of prime spectra associated with a non-commutative ring homomorphism $R \rightarrow S$. Our main result provides criteria for the adjointness of certain functors between the categories of Zariski closed subsets of Spec $R$ and $\operatorname{Spec} S$; these functors arise naturally from restriction and extension of scalars. When $R$ and $S$ are left Noetherian, adjointness occurs only for centralizing and 'nearly centralizing' homomorphisms.
\end{abstract}

Keywords: non-commutative ring; ring extension; prime ideal

2000 Mathematics subject classification: Primary 16D25; 16S20

Secondary

\section{Introduction}

One of the most elementary and well-known properties of non-commutative rings is the non-functoriality of their prime spectra: there is apparently no natural way of assigning, to an arbitrary ring homomorphism $R \rightarrow S$, a function from the prime spectrum of $S$ into the prime spectrum of $R$. Nevertheless, there is an extensive and deep literature presenting, among many other things, topological and geometric contexts for both non-commutative ring homomorphisms and their generalizations to certain functors between module-like categories. These contexts appear, for example, in the earlier publications $[\mathbf{1}],[\mathbf{5}],[\mathbf{1 8}],[\mathbf{2 0}]$ and $[\mathbf{1 9}]$, and the more recent ones $[\mathbf{2}],[\mathbf{1 0}],[\mathbf{1 2}],[\mathbf{1 1}],[\mathbf{1 4}],[\mathbf{1 3}]$ and $[\mathbf{1 5}]$. In the present paper we continue a discussion begun in $[\mathbf{1}, \S 4]$. We focus on topological properties of the correspondences of prime spectra associated with arbitrary homomorphisms involving left Noetherian rings or affine polynomial identity (PI) algebras.

1.1 .

To fix notation, equip the set Spec $R$ of prime ideals of a (not necessarily commutative) ring $R$ with the Zariski topology, by declaring the closed subsets to be those of the form

$$
V_{R}(X)=\{P \in \operatorname{Spec} R: P \supseteq X\}
$$


for $X \subseteq R$. Our specific intent in this paper is to carefully examine non-commutative generalizations of the following two trivially true but fundamentally important facts. If $f: R \rightarrow S$ is a commutative ring homomorphism, then

(1) the set map $\boldsymbol{r}: \operatorname{Spec} S \stackrel{P \mapsto f^{-1}(P)}{\longrightarrow}$ Spec $R$ is Zariski continuous, and

(2) $\boldsymbol{r}^{-1} V_{R}(X)=V_{S}(f(X))$.

1.2 .

Now let $f: R \rightarrow S$ be a homomorphism of non-commutative rings, and let

$$
\boldsymbol{r}: \operatorname{Spec} S \rightarrow \operatorname{Spec} R
$$

denote the correspondence assigning to each $P \in \operatorname{Spec} S$ the set of prime ideals of $R$ minimal over $f^{-1}(P)$. Adapting $[\mathbf{1}, \S 4]$, we will say that $\boldsymbol{r}$ is continuous provided

$$
\boldsymbol{r}^{[-1]} V:=\{P \in \operatorname{Spec} S: \boldsymbol{r} P \subseteq V\}
$$

is closed for all closed subsets $V$ of $\operatorname{Spec} R$.

It need not be true that $\boldsymbol{r}$ is continuous, even when $R$ and $S$ are Noetherian; see $\S 2.5$. Continuity does hold when $R$ and $S$ satisfy a polynomial identity; see [1, 4.6v] and (2.10).

One generalization of (2) might require that

$$
\boldsymbol{r}^{[-1]} V_{R}(X)=V_{S}(f(X))
$$

for all $X \subseteq S$. But it is easy to show that $r$ can be continuous while not satisfying this hypothesis; see $\S 2.4$ (iii). Another possible generalization is

$\left(2^{\prime}\right)$ for all ideals $I$ of $R$,

$$
\boldsymbol{r}^{[-1]} V_{R}(I)=V_{S}\left(I^{S}\right), \quad \text { where } I^{S}:=\operatorname{ann}_{S}(S / S f(I)) .
$$

It follows, for example, from $\S 3.12$ that $\left(2^{\prime}\right)$ is also strictly stronger than $\left(1^{\prime}\right)$. However, condition $\left(2^{\prime}\right)$ will be useful in our 'point-free' approach, described next.

\section{3 .}

Let SPEC $R$ denote the category whose objects are the Zariski closed subsets of Spec $R$ and whose morphisms are the inclusions; similarly define SPEC $S$. In $\S 5$ we consider the functors

$$
\lambda: \operatorname{SPEC} S \stackrel{V_{S}(J) \mapsto V_{R}\left(f^{-1}(J)\right)}{\longrightarrow} \operatorname{SPEC} R \text { and } \rho: \operatorname{SPEC} R \stackrel{V_{R}(I) \mapsto V_{S}\left(I^{S}\right)}{\longrightarrow} \operatorname{SPEC} R,
$$

where $I$ is a semiprime ideal of $R$ and $J$ is a semiprime ideal of $S$. When $R$ and $S$ are commutative, it is easy to check that $\lambda$ is left adjoint to $\rho$; this adjointness amounts, essentially, to a reformulation of (2). 
In our main result, Theorem 3.5, we give precise criteria for $\lambda$ to be a left adjoint to $\rho$, under certain hypotheses (satisfied by left Noetherian rings and affine PI algebras); in particular, this adjointness holds if and only if $\boldsymbol{r}$ is a single-valued continuous function (allowing for a slight abuse of notation) and $\left(2^{\prime}\right)$ holds. When $S$ is left Noetherian, further equivalent conditions are given, amounting to a 'nearly centralizing' property. The moral is that, other than for centralizing extensions, this adjointness is a rare occurrence.

\section{4 .}

In the approach to non-commutative algebraic geometry in $[\mathbf{1 1}, \mathbf{1 7}]$, the ring homomorphism $f: R \rightarrow S$ provides only one example of an affine map between affine noncommutative spaces. Indeed, some of our analysis below can be formulated for more general morphisms between non-commutative spaces, and a greater portion can be restated for the setting in which the homomorphism $f: R \rightarrow S$ is replaced by an appropriate $R-S$-bimodule. While a few of the definitions and preliminary results in this paper are presented within this broader context, we leave a more complete generalization to the interested reader. Recent studies on non-commutative ring homomorphisms (and generalizations) from this point of view include [13-15].

\section{5 .}

Our emphasis on categories of closed, rather than open, subsets of topological spaces is a matter of convenience and personal preference. All of the results and observations below have dual versions involving the categories of open subsets of topological spaces, with inclusions again providing the morphisms.

\subsection{Conventions and notation}

(i) Let $A$ be a ring. We will always assume that the Zariski topology has been applied to $\operatorname{Spec} A$, and we will continue to use the notation SPEC as in $\S 1.3$. If $I$ is an ideal of $A$, we will use $\sqrt{I}$ to denote the prime radical of $I$, and if $U$ is a set of prime ideals in $A$, we will use $I(U)$ to denote their intersection; note that $V_{A}(I(U))$ is the closure of $U$ in $\operatorname{Spec} A$.

(ii) Let $A$ and $B$ be rings. We will use ${ }_{A} M$ as an abbreviation for 'the left $A$-module $M^{\prime}$ '. We will similarly use $M_{A}$ for right $A$-modules and ${ }_{A} M_{B}$ for $A$ - $B$-bimodules. We will use $\operatorname{ann}_{A} M$ to denote the annihilator of ${ }_{A} M$ and ann $M_{A}$ to denote the annihilator of $M_{A}$. The category of left $A$-modules will be denoted $\operatorname{Mod} A$.

(iii) The reader is referred to $[\mathbf{6}, \mathbf{9}]$ for further ring-theoretic background information.

\section{Continuous correspondences}

In this section we consider ring homomorphisms and continuity. This discussion can be regarded as a continuation of $[\mathbf{1}, \S 4]$, where correspondences between the maximal spectra of affine PI algebras are considered.

Throughout this section, $f: R \rightarrow S$ will be a homomorphism of rings. 
2.1.

(i) Let $X$ and $Y$ be sets. By a correspondence $\boldsymbol{c}: X \rightarrow Y$ we mean a function from $X$ into the set of subsets of $Y$. Following common practice, we will define

$$
c U:=\bigcup_{u \in U} c u \quad \text { and } \quad c^{-1} V:=\{u \in U: c u \cap V \neq \emptyset\}
$$

for subsets $U$ of $X$ and $V$ of $Y$. However, it will be more convenient for our purposes to use the following generalization of the inverse of a function,

$$
c^{[-1]} V:=\{u \in U: c u \subseteq V\} .
$$

Note that $X-c^{-1} V=c^{[-1]}(Y-V)$. Also,

$$
\boldsymbol{c}^{[-1]} \boldsymbol{c} U \supseteq U, \quad \boldsymbol{c} \boldsymbol{c}^{[-1]} V \subseteq V, \quad \boldsymbol{c} U \subseteq \boldsymbol{c} U^{\prime} \quad \text { and } \quad \boldsymbol{c}^{[-1]} V \subseteq \boldsymbol{c}^{[-1]} V^{\prime},
$$

for all $U \subseteq U^{\prime} \subseteq X$ and $V \subseteq V^{\prime} \subseteq Y$.

(ii) Let $X$ and $Y$ be topological spaces. Following $[\mathbf{1}, \S 4]$, we will say that the correspondence $\boldsymbol{c}: X \rightarrow Y$ is continuous provided $\boldsymbol{c}^{-1} W$ is open for all open subsets $W$ of $Y$, or, equivalently, provided $c^{[-1]} Z$ is closed for all closed subsets $Z$ of $Y$.

\section{2 .}

The correspondences of spectra of interest to us appear within the following more general framework. Let $\alpha: \operatorname{Mod} B \rightarrow \operatorname{Mod} A$ be a covariant functor, for rings $A$ and $B$. Given an ideal $J$ of $B$, set

$$
J^{\alpha}:=\operatorname{ann}_{A} \alpha(B / J) .
$$

We obtain a correspondence $\boldsymbol{r}(\alpha): \operatorname{Spec} B \rightarrow \operatorname{Spec} A$, sending each $P \in \operatorname{Spec} B$ to the set of prime ideals of $A$ minimal over $P^{\alpha}$. (It may be the case that $P^{\alpha}=A$, in which case $\boldsymbol{r}(\alpha) P$ will be empty. However, using Zorn's lemma, if $J$ is an ideal of $A$ contained within at least one $Q \in \operatorname{Spec} A$, then there exists a $Q^{\prime} \in \operatorname{Spec} A$ such that $Q^{\prime} \subseteq Q$ and such that $Q^{\prime}$ is minimal over $J$.)

\section{3.}

Applying $\S 2.2$ to the restriction of scalars functor $\operatorname{Mod} S \rightarrow \operatorname{Mod} R$, we obtain the correspondence (which we will denote) $\boldsymbol{r}: \operatorname{Spec} S \rightarrow \operatorname{Spec} R$, sending each $P \in \operatorname{Spec} S$ to the non-empty set

$$
\left\{Q \in \operatorname{Spec} R: Q \text { is minimal over } f^{-1}(P)=\operatorname{ann}_{R}(S / P)\right\} .
$$

If $Q$ is a prime ideal of $R$, then $\boldsymbol{r}^{-1} Q$ is commonly referred to as the set of prime ideals of $S$ 'lying over' $Q$. 
2.4 .

Let $I$ be an ideal of $R$.

(i) Note that

$$
\boldsymbol{r}^{[-1]} V_{R}(I)=\left\{P \in \operatorname{Spec} S: \sqrt{f^{-1}(P)} \supseteq I\right\}
$$

(ii) When $R$ and $S$ are commutative, $\boldsymbol{r}$ is the continuous function from $\operatorname{Spec} S$ to $\operatorname{Spec} R$ mapping each prime ideal $P$ of $S$ to the prime ideal $f^{-1}(P)$ of $R$, and

$$
\boldsymbol{r}^{[-1]} V_{R}(I)=\boldsymbol{r}^{-1} V_{R}(I)=V_{S}(f(I)) .
$$

(iii) When $S$ is not commutative, the equality in (ii) need not hold. For example, set

$$
S=\left[\begin{array}{cc}
k & k \\
k & k
\end{array}\right], \quad R=\left\{\left[\begin{array}{cc}
\alpha & \beta \\
0 & \alpha
\end{array}\right]: \alpha, \beta \in k\right\} \subseteq S \quad \text { and } \quad I=\left[\begin{array}{cc}
0 & k \\
0 & 0
\end{array}\right] \subseteq R,
$$

where $k$ is a field. Let $f$ be the inclusion of $R$ in $S$. Then $\operatorname{Spec} S=\{0\}, \operatorname{Spec} R=$ $\{I\}, r$ is continuous, and

$$
\boldsymbol{r}^{[-1]} V_{R}(I)=\{0\} \neq \emptyset=V_{S}(f(I)) .
$$

(iv) When $\sqrt{f^{-1}(P)}$ is nilpotent modulo $f^{-1}(P)$ for all $P \in \operatorname{Spec} S$, the equality in (ii) can be replaced by

$$
\begin{aligned}
\boldsymbol{r}^{[-1]} V_{R}(I) & =\left\{P \in \operatorname{Spec} S: f^{-1}(P) \supseteq I^{t} \text { for some positive integer } t\right\} \\
& =\left\{P \in \operatorname{Spec} S: P \supseteq f(I)^{t} \text { for some positive integer } t\right\} \\
& =\bigcup_{t \geqslant 1} V_{S}\left(f(I)^{t}\right) .
\end{aligned}
$$

\section{5.}

We can see as follows that $\boldsymbol{r}$ need not be continuous, even when $R$ and $S$ are Noetherian.

Let $k$ be a field of characteristic zero, and suppose that $S$ has been chosen to be the enveloping algebra of $\mathfrak{s l}_{2}(k)$. Let $\{E, F, H\}$ be the standard $k$-basis for $\mathfrak{s l}_{2}(k) \subset S$ (see, for example, $[\mathbf{4}, \S 1.8])$, with $[H, E]=2 E,[H, F]=-2 F$ and $[E, F]=H$. Assume that $R=k\{E\} \subset S$ and that $f$ is the inclusion map. Let $I=\langle E\rangle$. It is well known that $R$ is a polynomial ring in $E$ and that $S$ is Noetherian. Moreover, if $P$ is the kernel of a finite-dimensional irreducible representation of $S$, then $I^{t} \subset P$ for some positive integer $t$. (This last assertion immediately follows, e.g. from $[4, \S 1.8]$.)

We can now see that $U=\boldsymbol{r}^{[-1]} V_{R}(I) \subset \operatorname{Spec} S$ contains the kernel of every finitedimensional irreducible representation of $S$. It is well known that the intersection of these kernels is zero. Therefore, $I(U)=0$, a prime ideal of $S$. However, the ideal 0 of $S$ cannot be contained in $U$, and so $U \neq V_{S}(I(U))$. Therefore, $U$ is not closed, and $f$ is not continuous. 
2.6.

Continuity does hold in the following commonly occurring special case. Suppose that $f^{-1}(P)$ is a semiprime ideal of $R$ for every prime ideal $P$ of $S$. (See, for example, $[\mathbf{9}$, Chapter 10] for settings in which this hypothesis holds.) Then, if $I$ is an ideal of $R$,

$$
\boldsymbol{r}^{[-1]} V_{R}(I)=\left\{P \in \operatorname{Spec} S: f^{-1}(P) \supseteq I\right\}=\{P \in \operatorname{Spec} S: P \supseteq f(I)\}=V_{S}(f(I)) .
$$

Hence $\boldsymbol{r}$ is continuous.

\section{7 .}

In the remainder of this section we establish continuity in the presence of a bound on Goldie ranks.

(i) Let $A$ be a ring for which every prime factor is left or right Goldie. Set

$$
\operatorname{Spec}_{n} A=\{P \in \operatorname{Spec} A: \operatorname{rank}(A / P) \leqslant n\},
$$

where 'rank' means 'Goldie rank' and where $n$ is a positive integer. Equip $\operatorname{Spec}_{n} A$ with the relative Zariski topology.

(ii) Suppose that all of the prime factors of $R$ and $S$ are left or right Goldie. Let $P \in \operatorname{Spec}_{n} S$. It follows from [21] that $\boldsymbol{r} P \in \operatorname{Spec}_{n} R$.

Lemma 2.1. Let $A$ be a subring of a prime left or right Goldie ring B. Suppose that the Goldie rank of $B$ is $t$, and let $N$ denote the prime radical of $A$. Then $N^{t}=0$.

Proof. Let $F$ be the Goldie quotient ring of $B$. By assumption, $F$ has length $t$ as a left $F$-module, and so there exists an $F$-A-bimodule composition series

$$
0=F_{0} \subset F_{1} \subset \cdots \subset F_{s}=F,
$$

for some $s \leqslant t$. For $1 \leqslant i \leqslant s$, set

$$
Q_{i}=\operatorname{ann}\left(F_{i} / F_{i-1}\right)_{A} .
$$

Then $F \cdot Q_{s} \cdots Q_{1}=0$, and it is easy to check that $Q_{1}, \ldots, Q_{s}$ are prime ideals of $A$. In particular, $Q_{s} \cdots Q_{1}=0$ in $A$, and so $N^{t} \subseteq N^{s}=0$.

Proposition 2.2. Let $n$ be a positive integer, and assume that all of the prime factors of $R$ and $S$ are left or right Goldie. Then $\boldsymbol{r}: \operatorname{Spec}_{n} S \rightarrow \operatorname{Spec}_{n} R$ is continuous.

Proof. Without loss of generality, we may assume that $R$ is a subring of $S$ and that $f$ is the inclusion map. Let $I$ be an ideal of $R$, and set $V=V_{R}(I)$. It now follows from Lemma 2.1, and our earlier observations, that

$$
\begin{aligned}
\left(\boldsymbol{r}^{[-1]}\left(V \cap \operatorname{Spec}_{n} R\right)\right) \cap \operatorname{Spec}_{n} S & =\left(\boldsymbol{r}^{[-1]} V\right) \cap \operatorname{Spec}_{n} S \\
& =\left\{P \in \operatorname{Spec}_{n} S: P \supseteq I^{n}\right\} \\
& =\left(V_{S}\left(I^{n}\right)\right) \cap \operatorname{Spec}_{n} S .
\end{aligned}
$$

The proposition follows. 
Corollary 2.3 (see $4.6 \mathrm{v}$ in [1]). If $S$ is a PI ring, then $\boldsymbol{r}$ is continuous.

Proof. Assume that $S$ is PI. It follows from Posner's theorem that every prime factor of $R$ and $S$ is Goldie. It follows from basic PI theory that there exists a finite upper bound for the Goldie ranks of the prime factors of $S$. The corollary now follows from Proposition 2.2.

\section{8 .}

In $[\mathbf{1}, 4.6 \mathrm{v}]$ it is noted that the correspondence $\boldsymbol{r}: \max S \rightarrow \max R$ is continuous when $R$ and $S$ are PI algebras affine over a field. However, the proof given there (in the last paragraph on p. 307) appears to be incorrect.

2.9 .

In $[\mathbf{1}, 4.7]$ it is shown that the homomorphism $f: R \rightarrow S$ can be chosen with the following properties:

(i) $R$ and $S$ are PI algebras affine over a field,

(ii) there exists a closed subset $V$ of $\operatorname{Spec} R$ for which $\boldsymbol{r}^{-1} V$ is not closed in $\operatorname{Spec} S$.

As noted in $[\mathbf{1}, 4.7]$, it follows that ' $\boldsymbol{r}^{-1}$ (open) is open' continuity does not imply ' $r^{-1}$ (closed) is closed' continuity.

\subsection{0 .}

We ask two questions.

(i) Must $\boldsymbol{r}$ be continuous when $S$ is FBN?

(ii) Must $\boldsymbol{r}$ be continuous when $S$ is finitely generated as an $R$-module?

\section{Adjointness}

Throughout this section, $f: R \rightarrow S$ will be a ring homomorphism, and $r$ will denote the correspondence from Spec $S$ to Spec $R$ described in $\S 2.3$. In our main result, Theorem 3.5, we determine - under additional hypotheses introduced in $\S 3.7$ - when adjointness holds for the functors, between SPEC $R$ and SPEC $S$, arising from restriction and extension of scalars.

We begin with some preliminaries on functors, correspondences and topological spaces.

\section{1 .}

Let $X$ be a topological space, and let Closed $X$ denote the category whose objects are the closed subsets of $X$ and whose morphisms are the inclusions. If $U$ is a subset of $X$, we will denote the closure of $U$ in $X$ by $\bar{U}$. 
3.2 .

Let $X$ and $Y$ be topological spaces.

(i) Let $\varphi$ be a covariant functor from Closed $X$ to $\operatorname{Closed} Y$, and let $\psi$ be a covariant functor from Closed $Y$ to Closed $X$. Then $\varphi$ is a left adjoint to $\psi$ exactly when

$$
\varphi U \subseteq V \Longleftrightarrow U \subseteq \psi V
$$

for all $U \in$ Closed $X$ and $V \in \operatorname{Closed} Y$. Now suppose that $\psi$ and $\psi^{\prime}$ are both right adjoints to $\varphi$, and let $V \in \operatorname{Closed} Y$. Then

$$
\psi V \subseteq \psi V \Longrightarrow \varphi \psi V \subseteq V \Longrightarrow \psi V \subseteq \psi^{\prime} V .
$$

Similarly, $\psi^{\prime} V \subseteq \psi V$. It follows that $\psi$ and $\psi^{\prime}$ must be the same functor.

(ii) Let $c: X \rightarrow Y$ be a (not necessarily continuous) correspondence. We obtain covariant functors

$$
\varphi^{c}: \text { Closed } X \stackrel{U \mapsto \overline{c U}}{\longrightarrow} \text { Closed } Y \text { and } \varphi_{c}: \text { Closed } Y \stackrel{V \mapsto \overline{c^{[-1]} V}}{\longrightarrow} \text { Closed } X .
$$

Moreover, $\varphi^{c}$ is a left adjoint to $\varphi_{c}$ exactly when

$$
c U \subseteq V \Longleftrightarrow U \subseteq \overline{c^{[-1]} V}
$$

for all closed subsets $U$ of $X$ and $V$ of $Y$. Consequently, if $\boldsymbol{c}$ is continuous, it immediately follows that $\varphi^{c}$ is a left adjoint to $\varphi_{c}$. Conversely, if $\varphi^{c}$ is a left adjoint to $\varphi_{c}$, then

$$
\overline{\boldsymbol{c}^{[-1]} V} \subseteq \overline{\boldsymbol{c}^{[-1]} V} \Longrightarrow \boldsymbol{c}\left(\overline{\boldsymbol{c}^{[-1]} V}\right) \subseteq V \Longrightarrow \overline{\boldsymbol{c}^{[-1]} V} \subseteq \boldsymbol{c}^{[-1]} \boldsymbol{c}\left(\overline{\boldsymbol{c}^{[-1]} V}\right) \subseteq \boldsymbol{c}^{[-1]} V .
$$

We conclude that $\varphi^{c}$ is a left adjoint to $\varphi_{c}$ if and only if $c$ is continuous.

3.3 .

We now introduce functors between spectra in a somewhat more general framework. Assume that $A$ and $B$ are rings, and that $\alpha: \operatorname{Mod} B \rightarrow \operatorname{Mod} A$ is a covariant functor. Recall the notation of $\S 2.2$.

(i) Following $\S 3.2$ (ii), we obtain the functors

$$
\varphi^{\boldsymbol{r}(\alpha)}: \operatorname{SPEC} B \rightarrow \operatorname{SPEC} A \text { and } \varphi_{\boldsymbol{r}(\alpha)}: \operatorname{SPEC} A \rightarrow \operatorname{SPEC} B .
$$

(ii) Suppose that $\alpha$ is right exact. Then the assignment $J \mapsto J^{\alpha}$ preserves inclusions, and thus induces a functor

$$
\theta^{\alpha}: \operatorname{SPEC} B \stackrel{V \mapsto V_{A}\left(I(V)^{\alpha}\right)}{\longrightarrow} \operatorname{SPEC} A .
$$


3.4.

Retain the notation of $\S 3.3$, and assume that there exists an $A-B$-bimodule $M$ such that $\alpha L=M \otimes_{B} L$, for each left $B$-module $L$. Recall, by Watts's theorem (see, for example, [16, IV.10.1]), that this assumption holds if and only if $\alpha$ possesses a right adjoint.

(i) Observe that

$$
J^{\alpha}=\operatorname{ann}_{A}(M / M . J)
$$

for all ideals $J$ of $B$.

(ii) Note, for ideals $J_{1}$ and $J_{2}$ of $B$, that

$$
J_{1}^{\alpha} J_{2}^{\alpha} \cdot M \subseteq J_{1}^{\alpha} \cdot M \cdot J_{2} \subseteq M \cdot J_{1} J_{2},
$$

and so $J_{1}^{\alpha} J_{2}^{\alpha} \subseteq\left(J_{1} J_{2}\right)^{\alpha}$.

(iii) Let $J$ be an ideal of $B$, and suppose that $Q$ is a prime ideal of $A$ containing $J^{\alpha}$. Using Zorn's lemma, we can choose an ideal $P$ of $B$ maximal such that $P \supseteq J$ and such that $P^{\alpha} \subseteq Q$; it follows from (ii) that $P$ must be prime. Therefore,

$$
Q \supseteq P^{\alpha} \supseteq(\sqrt{J})^{\alpha}, \quad \text { and so } \sqrt{J^{\alpha}} \supseteq(\sqrt{J})^{\alpha} \supseteq J^{\alpha} \text {. }
$$

It follows that

$$
\theta^{\alpha} V_{B}(J)=V_{A}\left(J^{\alpha}\right)
$$

for all ideals $J$ of $B$.

(iv) Let $J$ be an ideal of $B$, and set

$$
X=\boldsymbol{r}(\alpha) V_{B}(J) \subseteq \operatorname{Spec} A .
$$

If $Q \in X$, then $Q \supseteq J^{\alpha}$, and so $\bar{X} \subseteq V_{A}\left(J^{\alpha}\right)$. Conversely, choose $Q \in V_{A}\left(J^{\alpha}\right)$. As in (iii), there exists a prime ideal $P$ of $B$ such that $P \supseteq J$ and such that $Q \supseteq P^{\alpha}$. There then exists (by another Zorn's lemma argument) a prime ideal $Q^{\prime}$ of $A$ minimal over $P^{\alpha}$ such that $Q^{\prime} \subseteq Q$. Because $Q^{\prime} \in X$, we see that $Q \in \bar{X}$, and so

$$
\varphi^{r(\alpha)} V_{B}(J)=\bar{X}=V_{A}\left(J^{\alpha}\right)=\theta^{\alpha} V_{B}(J) .
$$

We see, in the present setting, that $\theta^{\alpha}$ and $\varphi^{r(\alpha)}$ are the same functor.

\section{5.}

Applying $\S 3.4$ to the restriction of scalars functor $\operatorname{Mod} S \rightarrow \operatorname{Mod} R$, we obtain the functor $\lambda$ : SPEC $S \rightarrow \operatorname{SPEC} R$, sending

$$
V_{S}(J) \mapsto V_{R}\left(f^{-1}(J)\right)
$$

for ideals $J$ of $S$. Again using $\S 3.4$, we see that $\lambda=\varphi^{r}$. 
3.6.

(i) For each ideal $I$ of $R$, set

$$
I^{S}=\operatorname{ann}_{S}(S / S f(I)) .
$$

Applying $\S 3.4$ to the extension of scalars functor $\operatorname{Mod} R \rightarrow \operatorname{Mod} S$, we now obtain the functor $\rho: \operatorname{SPEC} R \rightarrow \operatorname{SPEC} S$, sending

$$
V_{R}(I) \mapsto V_{S}\left(I^{S}\right)
$$

for ideals $I$ of $R$.

(ii) Suppose that $R$ and $S$ are commutative. Then $\boldsymbol{r}: \operatorname{Spec} S \rightarrow \operatorname{Spec} R$ is a continuous function, and, in the notation of $\S 3.4, \rho=\varphi_{r}$. Moreover, following $\S 3.2$ (ii) we see that $\lambda$ is a left adjoint to $\rho$.

3.7.

For the remainder of this section we will assume

(i) that all semiprime factors of $R$ and $S$ are left or right Goldie, and

(ii) that the prime radicals of all of the factors of $R$ and $S$ are nilpotent.

\section{8 .}

(i) The hypotheses in $\S 3.7$ hold, of course, when $R$ and $S$ are left or right Noetherian.

(ii) Suppose that $R$ and $S$ are each affine over a commutative Noetherian ring and satisfy a polynomial identity. Then $\S 3.7$ (i) follows from Posner's theorem, and $\S 3.7$ (ii) follows from $[3]$.

(iii) Let $I$ be an ideal of $R$ or $S$. It follows from $\S 3.7$ (i) that $\sqrt{I}$ is the intersection of finitely many prime ideals and then from $\S 3.7$ (ii) that $I$ contains a finite product of prime ideals. In particular, there are finitely many prime ideals minimal over $I$.

\section{9 .}

In Lemmas 3.1, 3.2 and 3.3 and in $\S 3.10$ we will further assume that $R$ is a subring of $S$ and that $f$ is inclusion.

\section{Lemma 3.1.}

(i) If $J$ is an ideal of $S$, then $\lambda V_{S}(J)=V_{R}(J \cap R)$.

(ii) If $I$ is an ideal of $R$, then $\rho V_{R}(I)=V_{S}\left(I^{S}\right)$. 


\section{Proof.}

(i) Let $J$ be an ideal of $S$. For sufficiently large $t$,

$$
(\sqrt{J} \cap R)^{t} \subseteq J \cap R \subseteq \sqrt{J} \cap R,
$$

and so

$$
V_{R}(J \cap R)=V_{R}(\sqrt{J} \cap R)=\lambda V_{S}(J) .
$$

(ii) Let $I$ be an ideal of $R$. By $\S 3.4$ (ii), for sufficiently large $t$,

$$
\left((\sqrt{I})^{S}\right)^{t} \subseteq\left((\sqrt{I})^{t}\right)^{S} \subseteq I^{S} \subseteq(\sqrt{I})^{S},
$$

and so

$$
V_{S}\left(I^{S}\right)=V_{S}\left((\sqrt{I})^{S}\right)=\rho V_{R}(I) .
$$

(The preceding two arguments are symmetrical — note that $J \cap R=\operatorname{ann}_{R}(S / S J)$, for ideals $J$ of $S$.)

\subsection{0 .}

We can now see, in the present situation, that $\lambda$ is a left adjoint to $\rho$ exactly when

$$
V_{S}(J) \subseteq V_{S}\left(I^{S}\right) \Longleftrightarrow V_{R}(J \cap R) \subseteq V_{R}(I),
$$

or, equivalently,

$$
I^{S} \subseteq \sqrt{J} \Longleftrightarrow I \subseteq \sqrt{J \cap R},
$$

for all ideals $I$ of $R$ and $J$ of $S$.

\section{Lemma 3.2.}

(i) Let $I$ be an ideal of $R$, let $J$ be an ideal of $S$, and suppose that $V_{R}(J \cap R) \subseteq V_{R}(I)$. Then $V_{S}(J) \subseteq V_{S}\left(I^{S}\right)$.

(ii) $\lambda$ is a left adjoint to $\rho$ if and only if

$$
V_{S}(J) \subseteq V_{S}\left(I^{S}\right) \Longrightarrow V_{R}(J \cap R) \subseteq V_{R}(I),
$$

for all ideals $I$ of $R$ and $J$ of $S$.

\section{Proof.}

(i) Since $I \subseteq \sqrt{J \cap R}$, there exists a positive integer $t$ such that $I^{t} \subseteq J \cap R$. Hence $I^{t} S \subseteq J$, and so $\left(I^{t}\right)^{S} \subseteq J$. Therefore, by Lemma 3.1,

$$
V_{S}(J) \subseteq V_{S}\left(\left(I^{t}\right)^{S}\right)=\rho V_{R}\left(I^{t}\right)=\rho V_{R}(I)=V_{S}\left(I^{S}\right) .
$$

(ii) This follows immediately from (i) and $\S 3.10$. 
Lemma 3.3. The following are equivalent.

(i) $\lambda$ is a left adjoint to $\rho$.

(ii) For all $P \in \operatorname{Spec} S$ and $Q \in \operatorname{Spec} R$,

$$
Q^{S} \subseteq P \Longrightarrow Q \subseteq \sqrt{P \cap R} .
$$

Proof. It follows immediately from $\S 3.10$ that (i) $\Longrightarrow$ (ii).

Conversely, assume that (ii) is true, that $I$ is an ideal of $R$, that $J$ is an ideal of $S$, and that $V_{S}(J) \subseteq V_{S}\left(I^{S}\right)$. Then $I^{S} \subseteq \sqrt{J}$. Let $P$ be a prime ideal of $S$ minimal over $J$.

Using Zorn's lemma we can choose an ideal $Q$ of $R$ maximal among the ideals $I^{\prime}$ of $R$ for which $I^{\prime} \supseteq I$ and $I^{S} \subseteq P$. Because $P$ is prime, $\S 3.4$ (ii) ensures that $Q$ is prime. Therefore, by assumption, $Q \subseteq \sqrt{P \cap R}$, and so $I \subseteq \sqrt{P \cap R}$. Consequently, $I^{t} \subseteq P \cap R$ for a sufficiently large positive integer $t$.

Since $P$ was arbitrarily chosen among the finitely many prime ideals of $S$ minimal over $J$, we see that $I^{t} \subseteq \sqrt{J} \cap R$ for sufficiently large $t$. However, $(\sqrt{J} \cap R)^{t} \subseteq J \cap R$ for sufficiently large $t$, and so $I^{t} \subseteq J \cap R$ for sufficiently large $t$. Therefore, $I \subseteq \sqrt{J \cap R}$. Hence $V_{R}(J \cap R) \subseteq V_{R}(I)$, and it follows from Lemma 3.2 (ii) that (ii) $\Longrightarrow(\mathrm{i})$.

Lemma 3.4. Let $P \in \operatorname{Spec} S$. Then there exists a $Q \in \operatorname{Spec} R$ such that $Q$ is minimal over $P \cap R$ and such that $Q^{S} \subseteq P$.

Proof. We may assume, without loss of generality, that $P=0$. Next, by $\S 3.8$ (iii), there exists a prime ideal $\hat{Q}$ of $R$ such that $\hat{Q} \cdot N=0$ for some non-zero ideal $N$ of $R$. Choose a minimal prime ideal $Q$ of $R$ such that $Q \subseteq \hat{Q}$, and let $F$ denote the Goldie quotient ring of $S$. Since $F \cdot Q \cdot N=0$, and since ann $F_{S}=0$, we see that $F \cdot Q \neq F$. Consequently, $F / F Q$ is a non-zero $F-R$-bimodule. By Goldie's theorem, every left $S$ submodule of $F / F Q$ must have annihilator equal to $P$.

Now note that $F / F Q$ contains a non-zero $S-R$-bimodule factor of $S / S Q$. In particular, there exists an $S$-R-bimodule factor $B$ of $S / S Q$ with $\operatorname{ann}_{S} B=0$. Thus $Q^{S}=\operatorname{ann}_{S}(S / S Q)=0$, and the lemma follows.

Theorem 3.5. Assume that $f: R \rightarrow S$ be a ring homomorphism, that all semiprime factors of $R$ and $S$ are left or right Goldie, and that the prime radicals of all of the factors of $R$ and $S$ are nilpotent.

(1) The following are equivalent.

(i) $\lambda$ is a left adjoint to $\rho$.

(ii) The canonical correspondence $\boldsymbol{r}: \operatorname{Spec} S \rightarrow \operatorname{Spec} R$ defined in $\S 2.3$ is a singlevalued continuous function, and

$$
\boldsymbol{r}^{[-1]} V_{R}(I)=V_{S}\left(I^{S}\right)
$$

for all ideals $I$ of $R$. 
(2) If $S$ is left Noetherian then (i), (ii) and the following are equivalent.

(iii) For each $Q \in \operatorname{Spec} R$ there is a positive integer $t$ such that $f(Q)^{t} S \subseteq S f(Q)$.

(iv) For each ideal $I$ of $R$ there is a positive integer $t$ such that $f(I)^{t} S \subseteq S f(I)$.

Proof. We may assume, without loss of generality, that $R$ is a subring of $S$ and that $f$ is inclusion.

(1) (i) $\Longrightarrow$ (ii). Let $P \in \operatorname{Spec} S$. By Lemma 3.4, we can choose $Q \in \operatorname{Spec} R$ such that $Q$ is minimal over $P \cap R$ and such that $Q^{S} \subseteq P$. By $\S 3.10, Q \subseteq \sqrt{P \cap R}$, and so $Q=\sqrt{P \cap R}$. Hence $\boldsymbol{r} P=\{Q\}$, and $\boldsymbol{r}$ is a single-valued function.

Now, let $I$ be an ideal of $R$, and note that $P \in \boldsymbol{r}^{[-1]} V_{R}(I)$ if and only if $I \subseteq \sqrt{P \cap R}$. Hence, by $\S 3.10, \boldsymbol{r}^{[-1]} V_{R}(I)=V_{S}\left(I^{S}\right)$. In particular, $\boldsymbol{r}$ is continuous.

(ii) $\Longrightarrow$ (i). Assume that $P \in \operatorname{Spec} S$, that $Q \in \operatorname{Spec} R$, and that $Q^{S} \subseteq P$. In other words, $P \in V_{S}\left(Q^{S}\right)$. By hypothesis, $V_{S}\left(Q^{S}\right)=\boldsymbol{r}^{[-1]} V_{R}(Q)$, and hence $P \in$ $\boldsymbol{r}^{[-1]} V_{R}(Q)$. Therefore, $\boldsymbol{r} P \subseteq V_{R}(Q)$, and so $Q \subseteq \sqrt{P \cap R}$. It now follows from Lemma 3.3 that $\lambda$ is a left adjoint to $\rho$.

(2) Assume that $S$ is left Noetherian.

(i) $\Longrightarrow$ (iii). Suppose that $S / S Q \neq 0$; the desired conclusion immediately holds true otherwise. Next, since $S$ is left Noetherian, there exists a series of $S-R$-bimodules,

$$
0=M_{0} \subset M_{1} \subset \cdots \subset M_{n}=S / S Q,
$$

such that, for each $1 \leqslant i \leqslant n$,

$$
P_{i}=\operatorname{ann}_{S}\left(M_{i} / M_{i-1}\right) \in \operatorname{Spec} S
$$

(see, for example, $[6,2.13]$ ). In particular, $Q^{S}$ is contained in each of $P_{1}, \ldots, P_{n}$. In view of $\S 3.10$, it now follows from our assumptions that $Q \subseteq \sqrt{P_{i} \cap R}$, for $1 \leqslant i \leqslant n$. Therefore, for sufficiently large $t, Q^{t} \subseteq P_{1} \cdots P_{n}$. Consequently, $Q^{t} \cdot(S / S Q)=0$, and so $Q^{t} S \subseteq S Q$.

(iii) $\Longrightarrow$ (i). Assume that $Q \in \operatorname{Spec} R$, that $P \in \operatorname{Spec} S$, and that $Q^{S} \subseteq P$. Choose $t$ such that $Q^{t} S \subseteq S Q$. Then $S Q^{t} S \subseteq S Q$, and so $S Q^{t} S \subseteq Q^{S}$. Hence, $Q^{t} \subseteq$ $\left(S Q^{t} S\right) \cap R \subseteq P \cap R$. Therefore, $Q \subseteq \sqrt{P \cap R}$. By Lemma 3.3, $\lambda$ is a left adjoint to $\rho$.

(iii) $\Longleftrightarrow$ (iv). Assume (iii), and let $I$ be an arbitrary ideal of $R$. Choose $Q_{1}, \ldots, Q_{n} \in$ Spec $R$ such that $\sqrt{I}=Q_{1} \cap \cdots \cap Q_{n}$ and such that $Q_{1} \cdots Q_{n} \subseteq I$. Then, by assumption, for a sufficiently large positive integer $t, I^{n} t S \subseteq S Q_{1} \cdots Q_{n} \subseteq S I$, and (iv) holds true. The converse is trivial. 


\subsection{1 .}

It is easy to see that conditions (iii) and (iv) of Theorem 3.5 are satisfied when the homomorphism $f: R \rightarrow S$ is centralizing (i.e. $S$ is generated as a left $R$-module by a set $X$ such that $r \cdot x=x \cdot r$ for all $r \in R$ and $x \in X)$. Non-centralizing homomorphisms for which Theorem 3.5 (iii), (iv) hold are more rare, although ring embeddings associated with nilpotent Lie superalgebras provide such examples (see $[\mathbf{7 , 8}]$ for details). We can view ring homomorphisms satisfying Theorem 3.5 (iv) as being 'nearly centralizing'.

\subsection{2 .}

It is not true that $\lambda$ is a left adjoint to $\rho$ if and only if $\boldsymbol{r}$ is a single-valued continuous function. To provide an easy illustration, let $k$ be a field of characteristic zero and let $S$ denote the first Weyl algebra over $k: S$ is generated by $x$ and $y$, subject only to the relation $y x-x y=1$. Let $R$ be the commutative polynomial ring $k[x]$, identified with the subalgebra of $S$ generated by $x$, and let $f$ denote the inclusion homomorphism.

Let $P$ denote the zero ideal of $S$. Then $\operatorname{Spec} S=\{P\}$ and $P \cap R \in \operatorname{Spec} R$. Hence $\boldsymbol{r}$ is a single-valued continuous function.

Now let $I$ be the ideal of $R$ generated by $x$. Then $S I=S x$ is a proper left ideal of $S$, and so $S / S I \neq 0$. Since $S$ is a simple ring, $I^{S}=0$. Also, $I^{S} \subset P$ and $I \nsubseteq \sqrt{P \cap R}$. Therefore, by $\S 3.10, \lambda$ is not a left adjoint to $\rho$.

Acknowledgements. The author's research was supported in part by NSF grants DMS-9970413 and DMS-0196236. I am grateful to F. Van Oystaeyen for helpful comments on the literature.

\section{References}

1. M. Artin And W. Schelter, Integral ring homomorphisms, Adv. Math. 39 (1981), 289-329.

2. M. Artin And J. J. Zhang, Noncommutative projective schemes, Adv. Math. 109 (1994), 228-287.

3. A. Braun, The nilpotency of the radical in a finitely generated PI ring, J. Alg. 89 (1984), 375-396.

4. J. DixmiER, Enveloping algebras (1996 printing of the 1977 English translation), Graduate Studies in Mathematics, vol. 11 (American Mathematical Society, Providence, RI, 1996).

5. J. S. Golan, Structure sheaves over a noncommutative ring, Lecture Notes in Pure and Applied Mathematics, vol. 56 (Dekker, New York, 1980).

6. K. R. Goodearl and R. B. Warfield JR, An introduction to noncommutative Noetherian rings, London Mathematical Society Student Texts, vol. 16 (Cambridge University Press, 1989).

7. E. S. Letzter, Prime and primitive ideals in enveloping algebras of solvable Lie superalgebras, in Abelian groups and noncommutative rings, pp. 237-255, Contemporary Mathematics, vol. 130 (American Mathematical Society, Providence, RI, 1992).

8. E. S. LETZTER, On the ring extensions arising from completely solvable Lie superalgebras, J. Alg. 146 (1992), 175-189.

9. J. C. McConnell And J. C. Robson, Noncommutative Noetherian rings (WileyInterscience, Chichester, 1987). 
10. J. NDirahisha And F. VAn Oystaeyen, Grothendieck representations of categories and canonical noncommutative topologies, K-Theory 25 (2002), 355-371.

11. A. L. Rosenberg, Noncommutative algebraic geometry and representations of quantized algebras, Mathematics and its Applications, vol. 330 (Kluwer, Dordecht, 1995).

12. A. L. Rosenberg, Noncommutative schemes, Compositio Math. 112 (1998), 93-125.

13. S. P. Smith, Subspaces of non-commutative spaces, Trans. Am. Math. Soc. 354 (2002), 2131-2171.

14. S. P. Sмiтh, Maps between non-commutative spaces, Trans. Am. Math. Soc. 356 (2004), 2927-2944.

15. S. P. Smith And J. J. Zhang, Fibers in Ore extensions, Alg. Representat. Theory 5 (2002), 411-431.

16. B. Stenström, Rings of quotients, Die Grundlehren der Mathematischen Wissenschaften, vol. 217 (Springer, 1975).

17. M. VAN DEN BERGH, Blowing up of non-commutative smooth surfaces, Memoirs of the American Mathematical Society, vol. 154 (American Mathematical Society, Providence, RI, 2001).

18. F. Van Oystaeyen, Prime spectra in non-commutative algebra, Lecture Notes in Mathematics, vol. 444 (Springer, 1975).

19. F. VAn Oystaeyen And A. Verschoren, Reflectors and localization, Lecture Notes in Pure and Applied Mathematics, vol. 41 (Dekker, New York, 1979).

20. F. VAn Oystaeyen And A. Verschoren, Noncommutative algebraic geometry, Lecture Notes in Mathematics, vol. 887 (Springer, 1981).

21. R. B. Warfield JR, Prime ideals in ring extensions, J. Lond. Math. Soc. (2) 28 (1983), 453-460. 